\title{
Factores de corrección a la resistencia a compresión de testigos de hormigón. Análisis crítico de las normas cubanas e internacionales
}

\section{Correction factors to the concrete cores compressive strenght. Critical analysis of the cuban and the international standards}

\author{
$\underline{\text { A. Fernández Domínguez }}{ }^{(*)}$, J. J. Howland Albear ${ }^{(*)}$
}

RESUMEN

La norma cubana NC 724:2015 sólo exige la aplicación de un factor de corrección a la resistencia a compresión de un testigo de hormigón, e incluye otros dos con carácter informativo, uno de los cuales presenta incongruencias. Por su parte, en normativas internacionales de referencia se exige la aplicación de hasta 4 factores de corrección. A partir del análisis crítico de las normativas internacionales son propuestos los factores de corrección que pudieran aparecer en una actualización de la NC 724. Finalmente se demuestra, utilizando una base de datos de testigos de 15 edificios de La Habana, que las diferencias que se obtienen en la resistencia de un testigo al aplicar los factores propuestos respecto a la práctica actual en Cuba son estadísticamente significativas, alcanzando un valor promedio del $15 \%$.

Palabras clave: probeta; testigo; resistencia a compresión; factor de corrección.

\section{ABSTRACT}

The cuban standard NC 724:2015 only demands the application of one correction factor to the compressive strength of concrete cores, and includes two more as an informative way, one of them with incongruities. On the other side, international standards demand the use of 4 correction factors. The critical analysis of the international standards allows proposing the correction factors that should appear on an upgrade of the NC 724. Finally using a database of concrete cores of 15 buildings in Havana, it's proven that the differences in the corrected compressive strength of the concrete cores, obtained when applying the propose correction factors and the ones obtained when applying the NC 274:2015 are statistically significant, reaching average values of $15 \%$.

Keywords: standard test specimen; concrete core; compressive strength; correction factor.

Nota: Los autores de este artículo proporcionaron a la revista Informes de la Construcción un anexo con los datos sobre 156 muestras de testigos de los 15 edificios en estudio que, por motivos de espacio, no puede ser reproducido. Este anexo está a disposición de los lectores que quieran consultarlo previa petición a: informes@ietcc.csic.es.

(*) Universidad Tecnológica de La Habana «José Antonio Echevarría», CUJAE; Calle 114, No.119901, e/ Ciclovía y Rotonda, Marianao, La Habana, Cuba.

Persona de contacto/Corresponding author: afernandezd@civil.cujae.edu.cu (A. Fernández Domínguez)

ORCID: http://orcid.org/oooo-0oo1-5792-3031 (A. Fernández Domínguez);

http://orcid.org/oooo-0002-8022-6645 (J. J. Howland Albear)

Cómo citar este artículo/Citation: Fernández Domínguez, A., Howland Albear, J. J. (2017). Factores de corrección a la resistencia a compresión de testigos de hormigón. Análisis crítico de las normas cubanas e internacionales. Informes de la Construcción, 69(547): e212, doi: http://dx.doi.org/10.3989/id53723

Copyright: (C) 2017 CSIC. Licencia / License: Salvo indicación contraria, todos los contenidos de la edición electrónica de Informes de la Construcción se distribuyen bajo una licencia de uso y distribución Creative Commons Attribution License (CC BY) Spain 3.o. 


\section{INTRODUCCIÓN}

La evaluación de la calidad del hormigón en una estructura existente constituye uno de los temas más investigados en la última década.

Diversas causas pueden motivar la evaluación del hormigón en la estructura. En obras en ejecución puede deberse a bajos resultados de resistencia a compresión obtenidos en las probetas; en obras terminadas puede estar asociado a la presencia de patologías en la estructura, al cambio de uso (aumento de la carga), entre otras.

La extracción y ensayo de testigos en combinación con ensayos no destructivos, como la esclerometría y el ultrasonido, constituye en la actualidad la manera más eficaz de evaluar la calidad del hormigón en la estructura, ya sea desde el punto de vista de resistencia a compresión como de durabilidad.

La resistencia a compresión que se obtiene al ensayar un testigo debe ser corregida, para tener en cuenta las diferencias que existen entre los testigos y probetas en aspectos como: la edad, las dimensiones, el estado de saturación en el momento del ensayo, la presencia de acero de refuerzo en su interior y el hecho de que los primeros son sometidos al proceso agresivo de extracción. También deben corregirse diferencias asociadas a propiedades del material, como la exudación.

Por otra parte, es evidente que un testigo, que representa al hormigón de una estructura, va a presentar resistencias más bajas que una probeta del mismo hormigón, por las diferencias marcadas en sus procesos de ejecución: vertido, compactación y curado, pero éstas no deben ser motivo de corrección alguna ya que se estaría desconociendo la realidad del hormigón en la estructura.

\section{ESPECIFICACIONES NORMATIVAS SOBRE LOS FACTORES DE CORRECCIÓN}

En la actualidad se manejan alrededor de 10 factores de corrección, de los cuales sólo 6 son referidos en las normativas internacionales de referencia del tema: ACI 214.4-10(1), BS EN 12504-1:2009(2), NBR 7680-1:2015(3), ASTM C42/C42M04(4), así como en la cubana NC 724:2015(5). Es importante resaltar que existen diferencias entre los factores de corrección que son tenidos en cuenta por una normativa u otra y que aquellos de aplicación generalizada presentan incluso valores diferentes en los distintos reglamentos técnicos, además en algunos casos los factores de corrección sólo son referidos en las normativas con carácter informativo. Los criterios sobre los factores de corrección de cada una de las normativas mencionadas son abordados a continuación. Los aspectos relacionados con otros factores de corrección que no están normalizados pueden ser encontrados en la literatura (6) (7) (8) (9).

\subsection{ACI 214.4-10}

Según el ACI 214.4-10(1) la resistencia a compresión obtenida del ensayo de un testigo $\left(f_{\text {test }}\right)$ debe ser corregida, empleando la ecuación 1.

$$
f_{\text {test,corr }}=F_{l / d} * F_{\text {dia }} * F_{m c}^{*} F_{d}^{*} f_{\text {test }}
$$

Donde $f_{\text {test,corr }}$ es la resistencia corregida de cada testigo y $F_{l / d}$, $F_{d i a}, F_{\text {hum }}$ y $F_{\text {dañ }}$ son factores que toman en cuenta efectos de esbeltez, diámetro, condiciones de humedad y daños durante la extracción. La Tabla 1 muestra valores promedios y coeficientes de variación para cada uno de esos factores reportados por Barlett y MacGregor en 1995 y asumidos por esta normativa.

\subsection{BS EN 12504-1:2009}

La normativa británica BS EN 12504-1:2009 (2) presenta en su anexo nacional factores de corrección a la resistencia a compresión de un testigo, según las dimensiones del testigo (factor de esbeltez) (ver ecuación 2) y la presencia de acero de refuerzo (ver ecuación 3) para ser aplicados como plantea la ecuación 4, si el objetivo es determinar la resistencia a compresión in situ.

$$
F_{h / d}=\frac{2}{1,5+d / h}
$$

\begin{tabular}{|c|c|c|c|}
\hline & Factor & Valor medio & $\begin{array}{l}\text { Coeficiente de } \\
\text { variación } V(\%)\end{array}$ \\
\hline \multirow{3}{*}{$F_{h / d}$. Esbeltez } & Tratamiento de la ASTM $\mathrm{C}_{42} / \mathrm{C}_{42} \mathrm{M}(4)^{*}$ & $1-\left(0,13-\alpha f_{\text {testigo }}\right)(2-h / d)^{2 * * *}$ & $2,5\left(2-\frac{h}{d}\right)^{2}$ \\
\hline & Sumergido $48 \mathrm{~h}$ & $1-\left(0,117-\alpha f_{\text {testigo }}\right)(2-h / d)^{2 * * *}$ & $2,5\left(2-\frac{h}{d}\right)^{2}$ \\
\hline & Secado al aire ${ }^{* *}$ & $1-\left(0,144-\alpha f c_{\text {testigo }}\right)(2-h / d)^{2 * * *}$ & $2,5\left(2-\frac{h}{d}\right)^{2}$ \\
\hline \multirow{3}{*}{$F_{d i a} \cdot$ Diámetro del testigo } & 2 in. $(50 \mathrm{~mm})$ & 1,06 & 11,8 \\
\hline & 4 in. $(100 \mathrm{~mm})$ & 1,00 & 0,0 \\
\hline & 6 in. $(150 \mathrm{~mm})$ & 0,98 & 1,8 \\
\hline \multirow{3}{*}{$\begin{array}{l}F_{h u m} \text {. Contenido de humedad } \\
\text { del testigo }\end{array}$} & Tratamiento de la ASTM $\mathrm{C}_{42} / \mathrm{C}_{42} \mathrm{M}(4)^{*}$ & 1,00 & 2,5 \\
\hline & Sumergido $48 \mathrm{~h}$ & 1,09 & 2,5 \\
\hline & Secado al aire ${ }^{* *}$ & 0,96 & 2,5 \\
\hline \multicolumn{2}{|l|}{$F_{d a n ̃ n}$. Daño por extracción } & 1,06 & 2,5 \\
\hline
\end{tabular}

Tabla 1. Factores de corrección a la resistencia a compresión de testigos (1).

* La ASTM C42/C42M-04(4) establece un tratamiento del testigo después de extraído y hasta ser ensayado.

** Secado al aire durante 7 días a temperatura ambiente y humedad relativa menor de $60 \%$.

*** La constante $\alpha=4,3 \times 10^{-4} \mathrm{MPa}^{-1}$. 


$$
\begin{gathered}
F_{a}=1+1,5 \times \sum_{i}\left(\frac{\phi_{b i}}{\phi_{b t}} \times \frac{r_{i}}{h}\right) \\
f_{\text {test }, \text { corr }}=F_{a} * F_{h / d} * f_{\text {test }}
\end{gathered}
$$

$F_{a}$ : factor de presencia de acero de refuerzo.

$\theta_{b i}$ : diámetro de la barra.

$\theta_{b t}$ : diámetro del testigo.

$r$ : distancia del eje de la barra al extremo más cercano del testigo.

$i$ : número de barras embebidas.

$h$ : longitud del testigo.

d: diámetro del testigo.

\subsection{NBR 7680-1:2015}

Esta normativa brasileña exige la aplicación de cuatro factores de corrección que deben ser aplicados según la ecuación 5. Ésta representa una nueva forma de aplicación de los factores de corrección respecto a la forma clásica multiplicativa que es empleada con frecuencia internacionalmente.

$$
f_{\text {test, corr }}=1 *\left(k_{1}+k_{2}+k_{3}+k_{4}\right) * f_{\text {test }}
$$

Los valores de los factores de corrección $k_{1}$ a $k_{4}$ se enumeran a continuación:

a) Relación de esbeltez ( $h / d) k_{1}$ (ver Tabla 2).

b) Efecto de daño por extracción en función del diámetro del testigo $\left(k_{2}\right)$ (ver Tabla 3$)$.

c) Dirección de extracción en relación a dirección de vertido del hormigón $\left(k_{3}\right)$.

Se plantea que para testigos extraídos de columnas, vigas, y paredes hormigonadas in situ $k_{3}=0,05$ mientras para testigos de losas $k_{3}=0$.

d) Efecto de humedad del testigo $\left(k_{4}\right)$.

Si los testigos son ensayados en estado saturado, después de $72 \mathrm{~h}$ sumergidos en agua, $k_{4}=0$; en cambio si son ensayados secos al aire (ambiente de laboratorio) durante 72 $\mathrm{h}, k_{4}=-0,04$.

\subsection{ASTM C42/C42M-O4}

Esta normativa sólo plantea la aplicación de un factor de corrección: el de esbeltez $\left(F_{h / d}\right)$, el cual asume los valores de la Tabla 4 en función de la relación altura-diámetro del testigo. El valor de resistencia a compresión corregido se determina entonces según la ecuación 6.

$$
f_{\text {test, }, \text { orr }}=f_{\text {test }} * F_{h / d}
$$

Tabla 4. Valores del factor de esbeltez según la ASTM C42/ C42M-04 (6) y la NC 724:2015 (10).

\begin{tabular}{|c|c|}
\hline $\boldsymbol{h} / \boldsymbol{d}$ & $\boldsymbol{F}_{\boldsymbol{h} / \boldsymbol{d}}$ según ASTM C42/C42M-o4 (6) \\
NC $_{\mathbf{7 2 4}} \mathbf{2 0 1 5}(\mathbf{1 0})$
\end{tabular}

\subsection{NC 724:2015}

La normativa cubana presenta en su anexo $\mathrm{C}$, con carácter obligatorio, la aplicación del factor de esbeltez $\left(F_{h / d}\right)$, y emplea los mismos valores de la Tabla 4.

Además en su anexo $\mathrm{C}$ presenta con carácter informativo dos factores de corrección $F_{1}$ y $F_{2}$.

F2 es un factor que considera la dirección de extracción del testigo con relación a la dirección del vertido del hormigón. Cuando la extracción del testigo es perpendicular a la dirección del vertido del hormigón $F 2$ adopta el valor de 1,05. Cuando la extracción del testigo y el vertido son en la misma dirección $F 2$ es 1,00.

F1 es un factor que considera las dimensiones del testigo y asume los valores de la Tabla 5 .

El valor de resistencia a compresión corregido de cada testigo se calcula entonces según la ecuación 7 .

$$
f_{\text {test,corr }}=f_{\text {test }} * F_{h / d} * F_{1} * F_{2}
$$

Tabla 5. Factor que considera las dimensiones del testigo (5).

\begin{tabular}{|l|c|c|c|c|}
\hline Dimensiones $(\mathbf{c m})$ & $20 \times 40$ & $15 \times 30$ & $10 \times 20$ & $5 \times 10$ \\
\hline Factor de corrección & 1,03 & 1 & 0,97 & 0,9 \\
\hline
\end{tabular}

\subsection{Discusión y conclusiones parciales}

El análisis de las normativas abordados de 2.1 a 2.5 evidencia que:

1. La normativa cubana NC 724:2015 (5) debe exigir un mayor número de factores de corrección a la resistencia a compresión del testigo. Hasta tanto no se lleven a cabo investigaciones propias, se cree que pueden asimilarse valores de otras normativas.

2. Debe mantenerse un factor de corrección por esbeltez $\left(F_{h / d}\right)$, pero en lugar de los valores actuales (Tabla 4), debe

Tabla 2. Valores de $k_{1}$ en función de la altura $(h)$ y el diámetro del testigo $(d)$ (3).

\begin{tabular}{|l|c|c|c|c|c|c|c|c|c|c|c|c|c|c|c|}
\hline $\boldsymbol{h} / \boldsymbol{d}$ & 2,00 & 1,88 & 1,75 & 1,63 & 1,5 & 1,41 & 1,33 & 1,25 & 1,21 & 1,18 & 1,14 & 1,11 & 1,07 & 1,04 & 1,00 \\
\hline $\boldsymbol{k}$ & 0,00 & $-0,01$ & $-0,02$ & $-0,03$ & $-0,04$ & $-0,05$ & $-0,06$ & $-0,07$ & $-0,08$ & $-0,09$ & $-0,10$ & $-0,11$ & $-0,12$ & $-0,13$ & $-0,14$ \\
\hline
\end{tabular}

Tabla 3. Valores de $k_{2}$ en función del diámetro del testigo (3).

\begin{tabular}{|l|c|c|c|c|c|}
\hline Diámetro del testigo (mm) & $\leq 25$ & $50^{*}$ & 75 & 100 & $\geq 150$ \\
\hline $\boldsymbol{k}_{2}$ & no permitido & 0,12 & 0,09 & 0,06 & 0,04 \\
\hline
\end{tabular}

* En este caso deben extraerse el doble de los testigos que para el resto de los diámetros. 
emplear la ecuación que propone el ACI 214.4-10 (1) para testigos sumergidos $48 \mathrm{~h}$ (ver Tabla 1), en concordancia con las exigencias sobre el tratamiento de los testigos de la NC 318:2015 (5). Dicho enfoque es mucho más completo, pues el factor depende además de la relación altura-diámetro de la resistencia a compresión del testigo.

3. El factor de la NC 724.2015 (10) que tiene en cuenta la dirección de extracción del testigo $(F 2)$ debe ser de aplicación obligatoria y además mantener sus valores actuales, los cuales están en concordancia con los exigidos por este concepto en la NBR 7680-1:2015 (3).

4. Debe eliminarse el factor de dimensiones $F 1$ de la NC 724:2015 (5), el cual presenta contradicciones respecto a los propuestos por otras normativas. Si se comparan los valores de la Tabla 5 con los valores del $F_{\text {dia }}$ de la Tabla 1 propuesto por el ACI 214.4-10 (1), saltan a la vista contradicciones. La aplicación de este factor está asociada entre otros fenómenos al llamado efecto volumen, que explica como testigos o probetas de hormigón con mayores diámetros y mayores volúmenes presentan menores valores de resistencia a compresión, pues tienen una mayor probabilidad de presentar oquedades y desperfectos en su interior. Esta teoría resulta totalmente válida si se trata de probetas, pero en el caso de testigos el daño que experimentan durante el proceso de extracción es tal que provoca un efecto totalmente contrario, o sea, pérdidas de resistencias a compresión mientras menor es el diámetro del testigo. En esta última afirmación se basan los valores de la Tabla 1 propuestos en el ACI 214.4-10 (1).

5. En su lugar debe exigirse el factor de daño por extracción propuesto por la NBR 7680-1:2015 (3) (ver Tabla 3) modificado para hacerlo compatible con la forma en que se aplican los factores en (5) (ver Tabla 6). Como puede observarse en la Tabla 7 la aplicación de ese factor tiene el mismo resultado que aplicar los factores de diámetro y de daño del ACI 214.4-10 (1).

6. Debe incluirse un factor de corrección por presencia de acero de refuerzo, ya que la NC 724:2015 (10) permite el ensayo de testigos con acero embebido en dirección longitudinal. Para este fin se cree que debe asumirse la ecuación 3 tomada de la BS EN 12504-1:2009 (2).

7. No existe consenso respecto al $F_{\text {hum }}$. La Tabla 8 muestra los diferentes valores planteados por normativas de referen- cia y algunos investigadores para $F_{\text {hum }}$, en la misma se observan las diferencias que existen en las distintas fuentes. Por tanto se cree que no se debe asumir valor alguno para dicho factor en una actualización de la NC 724.

8. Finalmente, el valor de resistencia a compresión obtenido del ensayo de un testigo $\left(f_{\text {test }}\right)$ debe ser corregido aplicando la ecuación 8 .

$$
f_{\text {test,corr }}=F_{h / d} * F_{d i r} * F_{d a \tilde{n}} * F_{a} * f_{\text {test }}
$$

Donde:

$f_{\text {testcorr }}$ es la resistencia a compresión corregida del testigo.

$f_{h / d}=1-\left(0,117-4,3 \times 10^{-4} * f_{\text {test }}\right)(2-h / d)^{2}$

$F_{d i r}=1,05$ para testigos extraídos en dirección perpendicular al hormigonado e igual a 1 para testigos extraídos en dirección paralela al hormigonado.

$F_{\text {dañ }}$ toma los valores de la Tabla 6 para testigos con diámetros que no aparezcan en esta tabla, el valor de $F_{d a \tilde{n}}$ se obtiene por interpolación lineal.

$F_{a}$ se determina según la ecuación 3 .

\section{MATERIALES Y MÉTODOS}

Una base de datos (facilitada a la revista Informes de la Construcción) conformada a partir informes de estudios de diagnóstico de diferentes obras en La Habana, llevados a cabo por dos entidades de la ciudad: la Empresa Nacional de Investigaciones Aplicadas (ENIA) y el Centro de Investigación y Desarrollo de la Construcción (CIDC), es utilizada en este apartado con el objetivo de cuantificar las diferencias que se producen en la resistencia a compresión corregida de un testigo, al aplicar los factores propuestos en 2.6 (ver ecuación 8) respecto a lo que exige hoy la NC 724: 2015 (6).

Fue detectado en dichos informes de diagnósticos que sólo se aplica al valor de resistencia a compresión obtenido de cada testigo, el $F_{h / d}$, que se establece con carácter obligatorio tanto en la NC 724:2015 (5) como en su antecesora la NC 318:2003 (10). En ningún caso se aplicaron los factores de corrección $F_{1}$ y $F_{2}$, que aparecen con carácter informativo tanto en la NC 724:2015 (5) como en la NC 318:2003 (10), no así en la NC

Tabla 6. Valores del $F_{\text {dañ }}$ propuesto para la actualización de la NC 724 .

\begin{tabular}{|l|c|c|c|c|c|}
\hline Diámetro del testigo $(\mathbf{m m})$ & $\leq 25$ & $50^{*}$ & 75 & 100 & $\geq 150$ \\
\hline$F_{\text {dañ }}$ & no permitido & 1,12 & 1,09 & 1,06 & 1,04 \\
\hline
\end{tabular}

Tabla 7. Comparación entre el enfoque de ACI 214.4-10 (1) y NBR 7680-1:2015 (3) sobre $F_{\text {dia }}$ y $F_{\text {dañ }}$

\begin{tabular}{|l|c|c|c|c|c|c|c|c|c|}
\cline { 2 - 11 } \multicolumn{1}{c|}{} & \multicolumn{3}{c|}{$\boldsymbol{F}_{\text {dia }}$} & \multicolumn{3}{c|}{$\boldsymbol{F}_{\text {dañ }}$} & \multicolumn{3}{c|}{ Efecto combinado } \\
(\%)
\end{tabular}

Tabla 8. Factores de humedad planteados diversas fuentes.

\begin{tabular}{|l|c|c|c|c|}
\hline \multicolumn{1}{|c|}{$\begin{array}{c}\text { Acondicionamiento } \\
\text { del testigo }\end{array}$} & ACI 214.4-10 & $\begin{array}{c}\text { Khoury } \\
(\mathbf{2 0 1 4})\end{array}$ & $\begin{array}{c}\text { NBR 7680- } \\
\mathbf{1 : 2 0 1 5}\end{array}$ & $\begin{array}{c}\text { Pérez Sánchez } \\
\text { (1998) }\end{array}$ \\
\hline Según ASTM & 1 & - & - & - \\
\hline Sumergido de 48 a 72 h & 1,09 & 1,09 & 1 & 1 \\
\hline Seco al aire de 3 a 7 días & 0,98 & 0,96 & 0,96 & 0,87 \\
\hline
\end{tabular}


54-122-1986 (11), la primera de las normativas del tema en el país encontrada en la literatura.

Por lo tanto, la comparación se realiza entre los valores de $f_{\text {test,corr }}$ que se obtienen al aplicar la ecuación 8, y al aplicar solamente el $F_{h / d}$ de la NC 724:2015 (5).

Algunas características de las obras que son caso de estudio se muestran en la Tabla 7. Todas las obras, ubicadas en La Habana, fueron escogidas al azar de un grupo numeroso de informes de estudios de diagnóstico. La muestra posee un total de 156 testigos distribuidos en 52 testigos extraídos de vigas (33\%), 10 de muros (6 \%), 59 de losas (39\%) y 35 de columnas (22\%).

La extracción de cada uno de estos testigos fue realizada conforme a las exigencias de las normas vigentes en cada época, ya sea la NC 54-122:1986 (11) o la NC 318:2003 (10). Cada uno de estos informes contiene además de la información reflejada en la Tabla 9 las dimensiones de cada uno de los testigos ensayados, así como su resistencia a compresión. Se asume que ninguno de los testigos ensayados presentaba acero de refuerzo en su interior, pues en ambas normativas no se permitía el ensayo de esta clase de testigos. De la misma forma se asume que los testigos fueron ensayados luego de estar sumergidos en agua $48 \mathrm{~h}$, la única forma de acondicionamiento de los testigos exigida en las mismas.

\section{RESULTADOS Y DISCUSIÓN}

Los valores de resistencia a compresión de cada testigo fueron corregidos empleando la ecuación $8\left(f_{\text {ec.8}}\right)$ y empleando solamente el $F_{h / d}$ de la NC 724:2015 (5) $\left(f_{724}\right)$ (ver Tablas 10, 11, 12 y 13), luego se determinó la relación entre los mismos $\left(f_{\text {ec. } 8} / f_{724}\right)$. Dichas relaciones fueron analizadas en el asistente estadístico STATGRAPHICS, donde se realizó una prueba de hipótesis cuya hipótesis nula $\left(H_{0}\right)$ fue que el promedio de las mismas era igual a uno $(\mu=1)$, mientras que la hipótesis alternativa fue que era mayor que uno $(\mu>1)$.

El resultado de la prueba de hipótesis para la muestra de tamaño 156 con valor promedio de 1,15 y una desviación estándar de 0,02 fue el rechazo de la hipótesis nula con un nivel de confianza del $99 \%$, lo que demostró que los valores de $f_{\text {ec. } 8}$ son significativamente superiores a los valores de $f_{724}$.

Cuantitativamente los valores de $f_{\text {ec. } 8}$ resultaron como promedio un $15 \%$ superiores en el caso de los testigos provenientes de columnas (ver Tabla 9); en el caso de vigas un $14 \%$ (ver Tabla 11), en losas un $10 \%$ (ver Tabla 12), mientras que en muros un $16 \%$ (ver Tabla 13). De manera general la diferencia en la resistencia a compresión corregida de los testigos fue de un $15 \%$ como promedio.

\section{CONCLUSIONES}

El trabajo realizado permite concluir que la actualización de la NC 724:2015 (5), en lo que respecta a los factores de corrección a la resistencia a compresión de testigos, resulta inminente. Fue demostrado con un $99 \%$ de confiabilidad que en la actualidad se está despreciando como promedio un $15 \%$ de la resistencia a compresión de los hormigones analizados en estructuras existentes. Hasta tanto no se realicen investigaciones en el país donde se determinen los factores de corrección para las particularidades de Cuba, se considera que deben ser asumidos los factores declarados en el apartado 2.6, los cuales son establecidos en las diferentes normativas internacionales de prestigio del tema.

\section{AGRADECIMIENTOS}

Se agradece a la Empresa Nacional de Investigaciones Aplicadas y al Centro de Investigación y Desarrollo de la Construcción por facilitar los informes de diagnósticos de las estructuras caso de estudio de este trabajo.

Tabla 9. Resumen sobre los datos más importantes de las 15 obras.

\begin{tabular}{|c|c|c|c|c|c|c|}
\hline $\mathbf{N} .^{\mathbf{o}}$ & Obra & $\begin{array}{c}\text { Año de } \\
\text { construcción }\end{array}$ & $\begin{array}{l}\text { Año de } \\
\text { estudio }\end{array}$ & $\begin{array}{l}\text { Elemento } \\
\text { ensayado }\end{array}$ & $\begin{array}{c}\text { Tamaño de } \\
\text { muestra }\end{array}$ & $\boldsymbol{f}_{\mathrm{cm}}(\mathrm{MPa})$ \\
\hline 1 & Almacén de Hemoderivados & 1943 & 2004 & Columna, viga, losa & 12 & 17,3 \\
\hline 2 & Calle N No. 336 & 1945 & 2002 & Viga, columna & 9 & 11,1 \\
\hline 3 & Almacenes Cubanacán & 1970 & 1999 & Columna, viga, losa & 19 & 13,9 \\
\hline 4 & Plaza de la Revolución José Martí & 1954 & 2006 & Losa & 4 & 19,1 \\
\hline 5 & Casa de Dulce María Loynaz & 1928 & 2002 & Losa & 4 & 6,8 \\
\hline 6 & Hotel Herradura & 1956 & 2000 & Viga, losa & 13 & 26,7 \\
\hline 7 & Edificio Malecón y F & 1967 & 2002 & Muro & 10 & 45,8 \\
\hline 8 & Muelle 8 del Mariel & 1979 & 2001 & Viga & 9 & 27,0 \\
\hline 9 & Edificio $7 \cdot{ }^{\mathrm{ma}}$ y 116 & 1964 & 2000 & Columna, viga & 12 & 6,4 \\
\hline 10 & Dirección Municipal del PCC Cárdenas & 1977 & 2002 & Losa & 3 & 27,3 \\
\hline 11 & Edificio Pentágono & 1956 & 1998 & Columna & 8 & 11,1 \\
\hline 12 & Basamento del Edificio de la UNAICC & 1927 & 2001 & Columna, viga & 6 & 9,1 \\
\hline 13 & Instituto Nacional de Oncología y Radiobiología & 1945 & 2004 & Losa & 12 & 21,8 \\
\hline 14 & Calixto García (Edificio Joaquín Albarrán) & 1914 & 2005 & Losa & 20 & 15,9 \\
\hline 15 & Hospital Pedro Borrás & 1935 & 2007 & Columna, viga & 15 & 14,7 \\
\hline
\end{tabular}


Tabla 10. Relación entre la resistencia a compresión de los testigos de columnas, aplicando los factores de corrección propuestos en 2.6 y el factor $F_{h / d}$ de la NC 724: 2015 (5).

\begin{tabular}{|c|c|c|c|c|c|c|c|c|c|c|c|c|c|c|}
\hline \multirow[b]{2}{*}{ Obra } & \multirow[b]{2}{*}{ Elemento } & \multirow[b]{2}{*}{$h(\mathrm{~cm})$} & \multirow[b]{2}{*}{$\begin{array}{c}d \\
(\mathrm{~cm})\end{array}$} & \multirow[b]{2}{*}{$\begin{array}{l}d_{\text {nominal }} \\
(\mathrm{mm})\end{array}$} & \multirow[b]{2}{*}{$h / d$} & \multirow[b]{2}{*}{$\begin{array}{c}\boldsymbol{f}_{\text {test }} \\
\text { (MPa) }\end{array}$} & \multicolumn{4}{|c|}{ Factores propuestos } & \multirow[b]{2}{*}{$\begin{array}{c}\boldsymbol{f}_{\text {ec.8 }} \\
\text { (Mpa) }\end{array}$} & \multirow[b]{2}{*}{$\begin{array}{c}F_{h / d} \mathrm{NC} \\
724\end{array}$} & \multirow[b]{2}{*}{$\begin{array}{c}\boldsymbol{f}_{724} \\
\text { (Mpa) }\end{array}$} & \multirow[b]{2}{*}{$\boldsymbol{f}_{\text {ec.8 }} / \boldsymbol{f}_{724}$} \\
\hline & & & & & & & $F_{h / d}$ & $F_{d a \tilde{n}}$ & $F_{a}$ & $\boldsymbol{F}_{d i r}$ & & & & \\
\hline 1 & Columna & 10,8 & 10 & 100 & 1,08 & 10,5 & 0,89 & 1,06 & 1 & 1,05 & 10,4 & 0,89 & 9,3 & 1,12 \\
\hline 1 & Columna & 15 & 10 & 100 & 1,50 & 10,3 & 0,97 & 1,06 & 1 & 1,05 & 11,1 & 0,96 & 9,9 & 1,12 \\
\hline 1 & Columna & 18 & 10 & 100 & 1,80 & 18,6 & 1,00 & 1,06 & 1 & 1,05 & 20,7 & 0,98 & 18,2 & 1,14 \\
\hline 2 & Columna & 12,4 & 6,95 & 70 & 1,78 & 10,4 & 0,99 & 1,096 & 1 & 1,05 & 11,8 & 0,98 & 10,2 & 1,16 \\
\hline 2 & Columna & 13,8 & 6,95 & 70 & 1,99 & 9,9 & 1,00 & 1,096 & 1 & 1,05 & 11,4 & 1,00 & 9,9 & 1,15 \\
\hline 2 & Columna & 13,7 & 6,95 & 70 & 1,97 & 8,5 & 1,00 & 1,096 & 1 & 1,05 & 9,8 & 1,00 & 8,5 & 1,15 \\
\hline 5 & Columna & 13,6 & 6,8 & 70 & 2,00 & 12,9 & 1,00 & 1,096 & 1 & 1,05 & 14,8 & 1,00 & 12,9 & 1,15 \\
\hline 5 & Columna & 13,6 & 6,8 & 70 & 2,00 & 12,9 & 1,00 & 1,096 & 1 & 1,05 & 14,8 & 1,00 & 12,9 & 1,15 \\
\hline 5 & Columna & 11,7 & 6,8 & 70 & 1,72 & 13,8 & 0,99 & 1,096 & 1 & 1,05 & 15,7 & 0,98 & 13,5 & 1,16 \\
\hline 5 & Columna & 11,7 & 6,8 & 70 & 1,72 & 20,4 & 0,99 & 1,096 & 1 & 1,05 & 23,2 & 0,98 & 20,0 & 1,16 \\
\hline 5 & Columna & 11,5 & 6,8 & 70 & 1,69 & 16,8 & 0,99 & 1,096 & 1 & 1,05 & 19,1 & 0,98 & 16,5 & 1,16 \\
\hline 5 & Columna & 11,5 & 6,8 & 70 & 1,69 & 11,0 & 0,99 & 1,096 & 1 & 1,05 & 12,5 & 0,98 & 10,8 & 1,16 \\
\hline 13 & Columna & 14 & 7 & 70 & 2,00 & 6,7 & 1,00 & 1,096 & 1 & 1,05 & 7,7 & 1,00 & 6,7 & 1,15 \\
\hline 13 & Columna & 14 & 7 & 70 & 2,00 & 5,6 & 1,00 & 1,096 & 1 & 1,05 & 6,4 & 1,00 & 5,6 & 1,14 \\
\hline 13 & Columna & 14 & 7 & 70 & 2,00 & 8,7 & $1, \mathrm{OO}$ & 1,096 & 1 & 1,05 & 10,0 & 1,00 & 8,7 & 1,15 \\
\hline 13 & Columna & 14 & 7 & 70 & 2,00 & 6,0 & 1,00 & 1,096 & 1 & 1,05 & 6,9 & 1,00 & 6,0 & 1,15 \\
\hline 13 & Columna & 14 & 7 & 70 & 2,00 & 7,8 & 1,00 & 1,096 & 1 & 1,05 & 9,0 & 1,00 & 7,8 & 1,15 \\
\hline 13 & Columna & 14 & 7 & 70 & 2,00 & 4,6 & 1,00 & 1,096 & 1 & 1,05 & 5,3 & 1,00 & 4,6 & 1,15 \\
\hline 15 & Columna & 22,8 & 12,4 & 125 & 1,84 & 12,6 & 1,00 & 1,05 & 1 & 1,05 & 13,9 & 0,99 & 12,5 & 1,11 \\
\hline 15 & Columna & 24,6 & 12,4 & 125 & 1,98 & 12,9 & 1,00 & 1,05 & 1 & 1,05 & 14,2 & 1,00 & 12,9 & 1,10 \\
\hline 15 & Columna & 24,6 & 12,4 & 125 & 1,98 & 10,5 & 1,00 & 1,05 & 1 & 1,05 & 11,6 & 1,00 & 10,5 & 1,10 \\
\hline 15 & Columna & 24,4 & 12,4 & 125 & 1,97 & 8,8 & 1,00 & 1,05 & 1 & 1,05 & 9,7 & 1,00 & 8,8 & 1,10 \\
\hline 15 & Columna & 24,6 & 12,4 & 125 & 1,98 & 13,4 & 1,00 & 1,05 & 1 & 1,05 & 14,8 & 1,00 & 13,4 & 1,10 \\
\hline 15 & Columna & 24,9 & 12,4 & 125 & 2,01 & 11,9 & 1,00 & 1,05 & 1 & 1,05 & 13,1 & 1,00 & 11,9 & 1,10 \\
\hline 15 & Columna & 24,6 & 12,4 & 125 & 1,98 & 9,6 & 1,00 & 1,05 & 1 & 1,05 & 10,6 & 1,00 & 9,6 & 1,10 \\
\hline 15 & Columna & 24,7 & 12,4 & 125 & 1,99 & 8,6 & 1,00 & 1,05 & 1 & 1,05 & 9,5 & 1,00 & 8,6 & 1,10 \\
\hline 16 & Columna & 15,4 & 10 & 100 & 1,54 & 8,4 & 0,97 & 1,06 & 1 & 1,05 & 9,1 & 0,96 & 8,1 & 1,12 \\
\hline 16 & Columna & 15,1 & 10 & 100 & 1,51 & 5,3 & 0,97 & 1,06 & 1 & 1,05 & 5,7 & 0,96 & 5,1 & 1,12 \\
\hline 16 & Columna & 10 & 10 & 100 & 1,00 & 8,3 & 0,87 & 1,06 & 1 & 1,05 & 8,0 & 0,87 & 7,2 & 1,11 \\
\hline 45 & Columna & 12 & 9,92 & 100 & 1,21 & 8,4 & 0,92 & 1,06 & 1 & 1,05 & 8,6 & 0,92 & 7,7 & 1,12 \\
\hline 45 & Columna & 12,8 & 9,9 & 100 & 1,29 & 13,6 & 0,94 & 1,06 & 1 & 1,05 & 14,2 & 0,93 & 12,6 & 1,13 \\
\hline 45 & Columna & 12,8 & 9,91 & 100 & 1,29 & 11,0 & 0,94 & 1,06 & 1 & 1,05 & 11,5 & 0,93 & 10,2 & 1,13 \\
\hline 45 & Columna & 12,1 & 9,92 & 100 & 1,22 & 9,7 & 0,92 & 1,06 & 1 & 1,05 & 9,9 & 0,92 & 8,9 & 1,11 \\
\hline 45 & Columna & 11,8 & 9,91 & 100 & 1,19 & 15,6 & 0,92 & 1,06 & 1 & 1,05 & 16,0 & 0,92 & 14,4 & 1,11 \\
\hline 45 & Columna & 12,6 & 9,91 & 100 & 1,27 & 10,4 & 0,93 & 1,06 & 1 & 1,05 & 10,8 & 0,93 & 9,7 & 1,11 \\
\hline & & & & & & & & & & & & Prom & edio & $\mathbf{1 , 1 5}$ \\
\hline
\end{tabular}


Tabla 11. Relación entre la resistencia a compresión de los testigos de vigas, aplicando los factores de corrección propuestos en 2.6 y el factor $F_{h / d}$ de la NC 724: 2015(5).

\begin{tabular}{|c|c|c|c|c|c|c|c|c|c|c|c|c|c|c|}
\hline \multirow[b]{2}{*}{ Obra } & \multirow[b]{2}{*}{ Elemento } & \multirow[b]{2}{*}{$h(\mathrm{~cm})$} & \multirow[b]{2}{*}{$\begin{array}{c}d \\
(\mathrm{~cm})\end{array}$} & \multirow[b]{2}{*}{$\begin{array}{c}d_{\text {nominal }} \\
(\mathrm{mm})\end{array}$} & \multirow[b]{2}{*}{$h / d$} & \multirow[b]{2}{*}{$\begin{array}{c}f_{\text {test }} \\
\text { (MPa) }\end{array}$} & \multicolumn{4}{|c|}{ Factores propuestos } & \multirow[b]{2}{*}{$\begin{array}{c}f_{\text {ec.8 }} \\
\text { (Mpa) }\end{array}$} & \multirow[b]{2}{*}{$\begin{array}{c}F_{h / d} \mathrm{NC} \\
724\end{array}$} & \multirow[b]{2}{*}{$\begin{array}{c}f_{724} \\
(\mathbf{M p a})\end{array}$} & \multirow[b]{2}{*}{$\boldsymbol{f}_{\text {ec. } 8} / \boldsymbol{f}_{724}$} \\
\hline & & & & & & & $F_{h / d}$ & $F_{d a \tilde{n}}$ & $F_{a}$ & $\boldsymbol{F}_{d i r}$ & & & & \\
\hline 1 & Viga & 10,3 & 10 & 100 & 1,03 & 19,6 & 0,89 & 1,06 & 1 & 1,05 & 19,4 & 0,88 & 17,2 & 1,13 \\
\hline 1 & Viga & 16,7 & 10 & 100 & 1,67 & 16,4 & 0,99 & 1,06 & 1 & 1,05 & 18,1 & 0,97 & 15,9 & 1,14 \\
\hline 1 & Viga & 20 & 10 & 100 & 2,00 & 19,3 & 1,00 & 1,06 & 1 & 1,05 & 21,5 & 1,00 & 19,3 & 1,11 \\
\hline 1 & Viga & 20 & 10 & 100 & 2,00 & 21,6 & 1,00 & 1,06 & 1 & 1,05 & 24,0 & 1,00 & 21,6 & 1,11 \\
\hline 1 & Viga & 16,3 & 10 & 100 & 1,63 & 26,3 & 0,98 & 1,06 & 1 & 1,05 & 28,7 & 0,97 & 25,5 & 1,13 \\
\hline 1 & Viga & 19,5 & 10 & 100 & 1,95 & 15,3 & 1,00 & 1,06 & 1 & 1,05 & 17,0 & 1,00 & 15,3 & 1,11 \\
\hline 2 & Viga & 13,9 & 6,95 & 70 & 2,00 & 14,3 & 1,00 & 1,096 & 1 & 1,05 & 16,5 & 1,00 & 14,3 & 1,15 \\
\hline 2 & Viga & 10,9 & 6,95 & 70 & 1,57 & 12,1 & 0,98 & 1,096 & 1 & 1,05 & 13,6 & 0,97 & 11,7 & 1,16 \\
\hline 2 & Viga & 14,2 & 6,95 & 70 & 2,04 & 10,3 & 1,00 & 1,096 & 1 & 1,05 & 11,9 & 1,00 & 10,3 & 1,16 \\
\hline 2 & Viga & 13,1 & 6,95 & 70 & 1,88 & 11,5 & 1,00 & 1,096 & 1 & 1,05 & 13,2 & 0,99 & 11,4 & 1,16 \\
\hline 2 & Viga & 9,2 & 6,95 & 70 & 1,32 & 10,8 & 0,94 & 1,096 & 1 & 1,05 & 11,7 & 0,94 & 10,2 & 1,15 \\
\hline 2 & Viga & 11,3 & 6,95 & 70 & 1,63 & 12,5 & 0,98 & 1,096 & 1 & 1,05 & 14,1 & 0,97 & 12,1 & 1,17 \\
\hline 5 & Viga & 8 & 6,8 & 70 & 1,18 & 18,3 & 0,92 & 1,096 & 1 & 1,05 & 19,4 & 0,91 & 16,7 & 1,16 \\
\hline 5 & Viga & 12 & 6,8 & 70 & 1,76 & 14,6 & 0,99 & 1,096 & 1 & 1,05 & 16,6 & 0,98 & 14,3 & 1,16 \\
\hline 5 & Viga & 12 & 6,8 & 70 & 1,76 & 14,6 & 0,99 & 1,096 & 1 & 1,05 & 16,6 & 0,98 & 14,3 & 1,16 \\
\hline 5 & Viga & 13 & 6,8 & 70 & 1,91 & 12,4 & 1,00 & 1,096 & 1 & 1,05 & 14,3 & 0,99 & 12,3 & 1,16 \\
\hline 5 & Viga & 12 & 6,8 & 70 & 1,76 & 16,5 & 0,99 & 1,096 & 1 & 1,05 & 18,8 & 0,98 & 16,2 & 1,16 \\
\hline 5 & Viga & 11 & 6,8 & 70 & 1,62 & 11,3 & 0,98 & 1,096 & 1 & 1,05 & 12,7 & 0,97 & 11,0 & 1,15 \\
\hline 5 & Viga & 11 & 6,8 & 70 & 1,62 & 21,5 & 0,98 & 1,096 & 1 & 1,05 & 24,2 & 0,97 & 20,9 & 1,16 \\
\hline 9 & Viga & 15 & 10 & 100 & 1,50 & 26,1 & 0,97 & 1,06 & 1 & 1,05 & 28,2 & 0,96 & 25,1 & 1,12 \\
\hline 9 & Viga & 15 & 10 & 100 & 1,50 & 30,9 & 0,97 & 1,06 & 1 & 1,05 & 33,4 & 0,96 & 29,7 & 1,12 \\
\hline 9 & Viga & 13,5 & 10 & 100 & 1,35 & 13,9 & 0,95 & 1,06 & 1 & 1,05 & 14,7 & 0,94 & 13,1 & 1,12 \\
\hline 9 & Viga & 17 & 10 & 100 & 1,70 & 24,7 & 0,99 & 1,06 & 1 & 1,05 & 27,2 & 0,98 & 24,2 & 1,12 \\
\hline 9 & Viga & 17 & 10 & 100 & 1,70 & 19,9 & 0,99 & 1,06 & 1 & 1,05 & 21,9 & 0,98 & 19,5 & 1,12 \\
\hline 9 & Viga & 17 & 10 & 100 & 1,70 & 25,0 & 0,99 & 1,06 & 1 & 1,05 & 27,5 & 0,98 & 24,5 & 1,12 \\
\hline 12 & Viga & 13,9 & 6,95 & 70 & 2,00 & 38,2 & 1,00 & 1,096 & 1 & 1,05 & 44,0 & 1,00 & 38,2 & 1,15 \\
\hline 12 & Viga & 13,9 & 6,95 & 70 & 2,00 & 21,8 & 1,00 & 1,096 & 1 & 1,05 & 25,1 & 1,00 & 21,8 & 1,15 \\
\hline 12 & Viga & 13,9 & 6,95 & 70 & 2,00 & 43,2 & 1,00 & 1,096 & 1 & 1,05 & 49,7 & 1,00 & 43,2 & 1,15 \\
\hline 12 & Viga & 8,7 & 6,95 & 70 & 1,25 & 27,4 & 0,93 & 1,096 & 1 & 1,05 & 29,3 & 0,93 & 25,5 & 1,15 \\
\hline 12 & Viga & 13,9 & 6,95 & 70 & 2,00 & 29,7 & 1,00 & 1,096 & 1 & 1,05 & 34,2 & 1,00 & 29,7 & 1,15 \\
\hline 12 & Viga & 13,9 & 6,95 & 70 & 2,00 & 15,8 & 1,00 & 1,096 & 1 & 1,05 & 18,2 & 1,00 & 15,8 & 1,15 \\
\hline 12 & Viga & 13,9 & 6,95 & 70 & 2,00 & 19,8 & 1,00 & 1,096 & 1 & 1,05 & 22,8 & 1,00 & 19,8 & 1,15 \\
\hline 12 & Viga & 11,2 & 6,95 & 70 & 1,61 & 18,1 & 0,98 & 1,096 & 1 & 1,05 & 20,4 & 0,97 & 17,6 & 1,16 \\
\hline 12 & Viga & 13,9 & 6,95 & 70 & 2,00 & 29,0 & 1,00 & 1,096 & 1 & 1,05 & 33,4 & 1,00 & 29,0 & 1,15 \\
\hline 13 & Viga & 14 & 7 & 70 & 2,00 & 7,4 & 1,00 & 1,096 & 1 & 1,05 & 8,5 & 1,00 & 7,4 & 1,15 \\
\hline 13 & Viga & 10,5 & 7 & 70 & 1,50 & 4,4 & 0,97 & 1,096 & 1 & 1,05 & 4,9 & 0,96 & 4,2 & 1,17 \\
\hline 13 & Viga & 14 & 7 & 70 & 2,00 & 5,3 & 1,00 & 1,096 & 1 & 1,05 & 6,1 & 1,00 & 5,3 & 1,15 \\
\hline 13 & Viga & 14 & 7 & 70 & 2,00 & 7,6 & 1,00 & 1,096 & 1 & 1,05 & 8,7 & 1,00 & 7,6 & 1,14 \\
\hline 13 & Viga & 13 & 7 & 70 & 1,86 & 7,4 & 1,00 & 1,096 & 1 & 1,05 & 8,5 & 0,99 & 7,3 & 1,16 \\
\hline 13 & Viga & 14 & 7 & 70 & 2,00 & 5,0 & 1,00 & 1,096 & 1 & 1,05 & 5,8 & 1,00 & 5,0 & 1,16 \\
\hline 16 & Viga & 15,5 & 10 & 100 & 1,55 & 12,1 & 0,97 & 1,06 & 1 & 1,05 & 13,1 & 0,96 & 11,6 & 1,13 \\
\hline 16 & Viga & 10,8 & 10 & 100 & 1,08 & 12,4 & 0,89 & 1,06 & 1 & 1,05 & 12,3 & 0,89 & 11,0 & 1,12 \\
\hline 16 & Viga & 15 & 10 & 100 & 1,50 & 8,3 & 0,97 & 1,06 & 1 & 1,05 & 9,0 & 0,96 & 8,0 & 1,13 \\
\hline 45 & Viga & 13,7 & 9,9 & 100 & 1,38 & 16,2 & 0,95 & 1,06 & 1 & 1,05 & 17,1 & 0,95 & 15,4 & 1,11 \\
\hline 45 & Viga & 13,4 & 9,91 & 100 & 1,35 & 14,9 & 0,95 & 1,06 & 1 & 1,05 & 15,8 & 0,94 & 14,0 & 1,13 \\
\hline 45 & Viga & 14,6 & 9,84 & 100 & 1,48 & 10,5 & 0,97 & 1,06 & 1 & 1,05 & 11,3 & 0,96 & 10,1 & 1,12 \\
\hline 45 & Viga & 14,3 & 9,91 & 100 & 1,44 & 15,6 & 0,96 & 1,06 & 1 & 1,05 & 16,7 & 0,95 & 14,8 & 1,13 \\
\hline 45 & Viga & 11 & 9,91 & 100 & 1,11 & 22,0 & 0,90 & 1,06 & 1 & 1,05 & 22,0 & 0,90 & 19,8 & 1,11 \\
\hline 45 & Viga & 13,7 & 9,9 & 100 & 1,38 & 12,3 & 0,95 & 1,06 & 1 & 1,05 & 13,0 & 0,95 & 11,7 & 1,11 \\
\hline 45 & Viga & 14,5 & 9,91 & 100 & 1,46 & 15,6 & 0,96 & 1,06 & 1 & 1,05 & 16,7 & 0,96 & 15,0 & 1,11 \\
\hline 45 & Viga & 10,6 & 9,72 & 100 & 1,09 & 22,6 & 0,90 & 1,06 & 1 & 1,05 & 22,6 & 0,89 & 20,1 & 1,12 \\
\hline 45 & Viga & 12,7 & 9,59 & 100 & 1,32 & 21,5 & 0,94 & 1,06 & 1 & 1,05 & 22,5 & 0,94 & 20,2 & 1,11 \\
\hline
\end{tabular}


Tabla 12. Relación entre la resistencia a compresión de los testigos de losas, aplicando los factores de corrección propuestos en $2.6 \mathrm{y}$ el factor $F_{h / d}$ de la NC 724: 2015 (5).

\begin{tabular}{|c|c|c|c|c|c|c|c|c|c|c|c|c|c|c|}
\hline \multirow[b]{2}{*}{ Obra } & \multirow[b]{2}{*}{ Elemento } & \multirow[b]{2}{*}{$h(\mathrm{~cm})$} & \multirow[b]{2}{*}{$\begin{array}{c}d \\
(\mathbf{c m})\end{array}$} & \multirow[b]{2}{*}{$\begin{array}{l}d_{\text {nominal }} \\
(\mathbf{m m})\end{array}$} & \multirow[b]{2}{*}{$h / d$} & \multirow[b]{2}{*}{$\begin{array}{c}f_{\text {test }} \\
\text { (MPa) }\end{array}$} & \multicolumn{4}{|c|}{ Factores propuestos } & \multirow[b]{2}{*}{$\begin{array}{c}f_{\text {ec.8 }} \\
\text { (Mpa) }\end{array}$} & \multirow[b]{2}{*}{$\begin{array}{c}F_{h / d} \mathrm{NC} \\
724\end{array}$} & \multirow[b]{2}{*}{$\begin{array}{c}f_{724} \\
(\text { Mpa) }\end{array}$} & \\
\hline & & & & & & & $\boldsymbol{F}_{h / d}$ & $\boldsymbol{F}_{d a \tilde{n}}$ & $\boldsymbol{F}_{\boldsymbol{a}}$ & $\boldsymbol{F}_{d i r}$ & & & & $\boldsymbol{f}_{\text {ec. } 8} / \boldsymbol{f}_{724}$ \\
\hline 1 & Losa & 13,5 & 10 & 100 & 1,35 & 17,8 & 0,95 & 1,06 & 1 & 1 & 17,9 & 0,94 & 16,7 & 1,07 \\
\hline 1 & Losa & 12,8 & 10 & 100 & 1,28 & 15,6 & 0,94 & 1,06 & 1 & 1 & 15,5 & 0,93 & 14,5 & 1,07 \\
\hline 1 & Losa & 12,2 & 10 & 100 & 1,22 & 15,9 & 0,93 & 1,06 & 1 & 1 & 15,7 & 0,92 & 14,6 & 1,08 \\
\hline 5 & Losa & 6,8 & 6,8 & 70 & 1,00 & 13,5 & 0,88 & 1,096 & 1 & 1 & 13,0 & 0,87 & 11,7 & 1,11 \\
\hline 5 & Losa & 6,8 & 6,8 & 70 & 1,00 & 9,1 & 0,87 & 1,096 & 1 & 1 & 8,7 & 0,87 & 7,9 & 1,10 \\
\hline 5 & Losa & 6,8 & 6,8 & 70 & 1,00 & 11,6 & 0,87 & 1,096 & 1 & 1 & 11,1 & 0,87 & 10,1 & 1,10 \\
\hline 5 & Losa & 8,8 & 6,8 & 70 & 1,29 & 8,8 & 0,94 & 1,096 & 1 & 1 & 9,1 & 0,93 & 8,2 & 1,11 \\
\hline 5 & Losa & 8 & 6,8 & 70 & 1,18 & 12,4 & 0,92 & 1,096 & 1 & 1 & 12,5 & 0,91 & 11,3 & 1,11 \\
\hline 5 & Losa & 8,3 & 6,8 & 70 & 1,22 & 11,6 & 0,92 & 1,096 & 1 & 1 & 11,7 & 0,92 & 10,7 & 1,09 \\
\hline 6 & Losa & 18,6 & 9,4 & 100 & 1,98 & 17,3 & 1,00 & 1,06 & 1 & 1 & 18,3 & 1,00 & 17,3 & 1,06 \\
\hline 6 & Losa & 16,2 & 9,4 & 100 & 1,72 & 18,3 & 0,99 & 1,06 & 1 & 1 & 19,2 & 0,98 & 17,9 & 1,07 \\
\hline 6 & Losa & 18,8 & 9,4 & 100 & 2,00 & 17,9 & 1,00 & 1,06 & 1 & 1 & 19,0 & 1,00 & 17,9 & 1,06 \\
\hline 6 & Losa & 18,5 & 9,4 & 100 & 1,97 & 22,7 & 1,00 & 1,06 & 1 & 1 & 24,1 & 1,00 & 22,7 & 1,06 \\
\hline 7 & Losa & 12 & 10 & 100 & 1,20 & 9,0 & 0,92 & 1,06 & 1 & 1 & 8,8 & 0,92 & 8,3 & 1,06 \\
\hline 7 & Losa & 12 & 10 & 100 & 1,20 & 4,9 & 0,92 & 1,06 & 1 & 1 & 4,8 & 0,92 & 4,5 & 1,07 \\
\hline 7 & Losa & 17,2 & 10 & 100 & 1,72 & 7,2 & 0,99 & 1,06 & 1 & 1 & 7,6 & 0,98 & 7,1 & 1,07 \\
\hline 7 & Losa & 12 & 10 & 100 & 1,20 & 6,0 & 0,92 & 1,06 & 1 & 1 & 5,9 & 0,92 & 5,5 & 1,07 \\
\hline 9 & Losa & 9 & 7 & 70 & 1,29 & 25,0 & 0,94 & 1,096 & 1 & 1 & 25,8 & 0,93 & 23,3 & 1,11 \\
\hline 9 & Losa & 11 & 10 & 100 & 1,10 & 30,1 & 0,91 & 1,06 & 1 & 1 & 29,0 & 0,89 & 26,8 & 1,08 \\
\hline 9 & Losa & 10 & 10 & 100 & 1,00 & 24,5 & 0,88 & 1,06 & 1 & 1 & 22,9 & 0,87 & 21,3 & 1,08 \\
\hline 9 & Losa & 10,7 & 10 & 100 & 1,07 & 19,4 & 0,89 & 1,06 & 1 & 1 & 18,3 & 0,89 & 17,3 & 1,06 \\
\hline 9 & Losa & 10 & 10 & 100 & 1,00 & 27,1 & 0,88 & 1,06 & 1 & 1 & 25,3 & 0,87 & 23,6 & 1,07 \\
\hline 9 & Losa & 10 & 10 & 100 & 1,00 & 43,8 & 0,89 & 1,06 & 1 & 1 & 41,3 & 0,87 & 38,1 & 1,08 \\
\hline 9 & Losa & 7 & 7 & 70 & 1,00 & 36,7 & 0,89 & 1,096 & 1 & 1 & 35,8 & 0,87 & 31,9 & 1,12 \\
\hline 14 & Losa & 10,3 & 6,95 & 70 & 1,48 & 27,0 & 0,97 & 1,096 & 1 & 1 & 28,7 & 0,96 & 25,9 & 1,11 \\
\hline 14 & Losa & 10 & 6,95 & 70 & 1,44 & 22,9 & 0,96 & 1,096 & 1 & 1 & 24,1 & 0,95 & 21,8 & 1,11 \\
\hline 14 & Losa & 8,7 & 6,95 & 70 & 1,25 & 32,0 & 0,93 & 1,096 & 1 & 1 & 32,6 & 0,93 & 29,8 & 1,09 \\
\hline 17 & Losa & 10,75 & 7,41 & 75 & 1,45 & 32,9 & 0,97 & 1,09 & 1 & 1 & 34,8 & 0,95 & 31,3 & 1,11 \\
\hline 17 & Losa & 10,51 & 7,41 & 75 & 1,42 & 21,3 & 0,96 & 1,09 & 1 & 1 & 22,3 & 0,95 & 20,2 & 1,10 \\
\hline 17 & Losa & 8,41 & 7,41 & 75 & 1,13 & 19,5 & 0,91 & 1,09 & 1 & 1 & 19,3 & 0,90 & 17,6 & 1,10 \\
\hline 17 & Losa & 10,35 & 7,41 & 75 & 1,40 & 15,3 & 0,96 & 1,09 & 1 & 1 & 16,0 & 0,95 & 14,5 & 1,10 \\
\hline 17 & Losa & 9,33 & 7,41 & 75 & 1,26 & 24,1 & 0,93 & 1,09 & 1 & 1 & 24,4 & 0,93 & 22,4 & 1,09 \\
\hline 17 & Losa & 9,53 & 7,41 & 75 & 1,29 & 21,3 & 0,94 & 1,09 & 1 & 1 & 21,8 & 0,93 & 19,8 & 1,10 \\
\hline 17 & Losa & 9,13 & 7,41 & 75 & 1,23 & 13,4 & 0,93 & 1,09 & 1 & 1 & 13,6 & 0,93 & 12,5 & 1,09 \\
\hline 17 & Losa & 9,71 & 7,41 & 75 & 1,31 & 25,5 & 0,94 & 1,09 & 1 & 1 & 26,1 & 0,94 & 24,0 & 1,09 \\
\hline 17 & Losa & 7,96 & 7,41 & 75 & 1,07 & 20,9 & 0,90 & 1,09 & 1 & 1 & 20,5 & 0,89 & 18,6 & 1,10 \\
\hline 17 & Losa & 8,16 & 7,41 & 75 & 1,10 & 30,1 & 0,91 & 1,09 & 1 & 1 & 29,9 & 0,89 & 26,8 & 1,12 \\
\hline 17 & Losa & 8,48 & 7,41 & 75 & 1,14 & 19,9 & 0,91 & 1,09 & 1 & 1 & 19,7 & 0,90 & 17,9 & 1,10 \\
\hline 17 & Losa & 8,27 & 7,5 & 75 & 1,10 & 17,7 & 0,90 & 1,09 & 1 & 1 & 17,4 & 0,89 & 15,8 & 1,10 \\
\hline 31 & Losa & 9,92 & 7 & 70 & 1,42 & 14,8 & 0,96 & 1,096 & 1 & 1 & 15,6 & 0,95 & 14,1 & 1,11 \\
\hline 31 & Losa & 10,04 & 6,91 & 70 & 1,45 & 14,4 & 0,96 & 1,096 & 1 & 1 & 15,2 & 0,95 & 13,7 & 1,11 \\
\hline 31 & Losa & 9,97 & 6,92 & 70 & 1,44 & 9,8 & 0,96 & 1,096 & 1 & 1 & 10,3 & 0,95 & 9,3 & 1,11 \\
\hline 31 & Losa & 9,48 & 6,93 & 70 & 1,37 & 9,3 & 0,95 & 1,096 & 1 & 1 & 9,7 & 0,94 & 8,7 & 1,11 \\
\hline 31 & Losa & 10,63 & 6,92 & 70 & 1,54 & 37,2 & 0,98 & 1,096 & 1 & 1 & 40,0 & 0,96 & 35,7 & 1,12 \\
\hline 31 & Losa & 11,24 & 7 & 70 & 1,61 & 35,6 & 0,98 & 1,096 & 1 & 1 & 38,2 & 0,97 & 34,5 & 1,11 \\
\hline 31 & Losa & 9,44 & 6,93 & 70 & 1,36 & 10,6 & 0,95 & 1,096 & 1 & 1 & 11,0 & 0,94 & 10,0 & 1,10 \\
\hline 31 & Losa & 10,9 & 6,92 & 70 & 1,58 & 10,0 & 0,98 & 1,096 & 1 & 1 & 10,7 & 0,97 & 9,7 & 1,10 \\
\hline 31 & Losa & 10,91 & 6,94 & 70 & 1,57 & 5,8 & 0,98 & 1,096 & 1 & 1 & 6,2 & 0,97 & 5,6 & 1,11 \\
\hline 31 & Losa & 10,04 & 6,93 & 70 & 1,45 & 6,9 & 0,96 & 1,096 & 1 & 1 & 7,3 & 0,95 & 6,6 & 1,11 \\
\hline 31 & Losa & 9,11 & 6,93 & 70 & 1,31 & 11,9 & 0,94 & 1,096 & 1 & 1 & 12,3 & 0,94 & 11,2 & 1,10 \\
\hline 31 & Losa & 11,34 & 6,93 & 70 & 1,64 & 10,9 & 0,98 & 1,096 & 1 & 1 & 11,7 & 0,97 & 10,6 & 1,10 \\
\hline 31 & Losa & 10,69 & 6,93 & 70 & 1,54 & 15,1 & 0,97 & 1,096 & 1 & 1 & 16,1 & 0,96 & 14,5 & 1,11 \\
\hline 31 & Losa & 10,62 & 6,95 & 70 & 1,53 & 13,7 & 0,97 & 1,096 & 1 & 1 & 14,6 & 0,96 & 13,2 & 1,11 \\
\hline 31 & Losa & 10,81 & 7 & 70 & 1,54 & 19,0 & 0,97 & 1,096 & 1 & 1 & 20,2 & 0,96 & 18,2 & 1,11 \\
\hline 31 & Losa & 11,39 & 6,94 & 70 & 1,64 & 20,1 & 0,98 & 1,096 & 1 & 1 & 21,6 & 0,97 & 19,5 & 1,11 \\
\hline 31 & Losa & 10,18 & 7 & 70 & 1,45 & 21,6 & 0,96 & 1,096 & 1 & 1 & 22,7 & 0,95 & 20,5 & 1,11 \\
\hline 31 & Losa & 8,98 & 6,93 & 70 & 1,30 & 22,3 & 0,94 & 1,096 & 1 & 1 & 23,0 & 0,94 & 21,0 & 1,10 \\
\hline 31 & Losa & 8,71 & 6,92 & 70 & 1,26 & 13,8 & 0,93 & 1,096 & 1 & 1 & 14,1 & 0,93 & 12,8 & 1,10 \\
\hline 31 & Losa & 8,9 & 6,94 & 70 & 1,28 & 15,1 & 0,94 & 1,096 & 1 & 1 & 15,6 & 0,93 & 14,0 & 1,11 \\
\hline
\end{tabular}

\begin{tabular}{l|l|} 
Promedio & $\mathbf{1 , 1 0}$ \\
\hline
\end{tabular} 
Tabla 13. Relación entre la resistencia a compresión de los testigos de muros, aplicando los factores de corrección propuestos en 2.6 y el factor $F_{h / d}$ de la NC 724: 2015 (5).

\begin{tabular}{|c|c|c|c|c|c|c|c|c|c|c|c|c|c|c|}
\hline \multirow[b]{2}{*}{ Obra } & \multirow[b]{2}{*}{ Elemento } & \multirow[b]{2}{*}{$h(\mathrm{~cm})$} & \multirow[b]{2}{*}{$\begin{array}{c}d \\
(\mathrm{~cm})\end{array}$} & \multirow[b]{2}{*}{$\begin{array}{l}d_{\text {nominal }} \\
(\mathrm{mm})\end{array}$} & \multirow[b]{2}{*}{$h / d$} & \multirow[b]{2}{*}{$\begin{array}{c}\boldsymbol{f}_{\text {test }} \\
\text { (MPa) }\end{array}$} & \multicolumn{4}{|c|}{ Factores propuestos } & \multirow[b]{2}{*}{$\begin{array}{c}\boldsymbol{f}_{\text {ec.8 }} \\
\text { (Mpa) }\end{array}$} & \multirow[b]{2}{*}{$\begin{array}{c}F_{h / d} \mathrm{NC} \\
724\end{array}$} & \multirow[b]{2}{*}{$\begin{array}{c}\boldsymbol{f}_{724} \\
(\mathbf{M p a})\end{array}$} & \multirow[b]{2}{*}{$\boldsymbol{f}_{\text {ec. } 8} / \boldsymbol{f}_{724}$} \\
\hline & & & & & & & $F_{h / d}$ & $F_{d a \tilde{n}}$ & $\boldsymbol{F}_{\boldsymbol{a}}$ & $F_{d i r}$ & & & & \\
\hline 11 & Muro & 9,8 & 6,95 & 70 & 1,41 & 44,5 & 0,96 & 1,096 & 1 & 1,05 & 49,2 & 0,95 & 42,3 & 1,16 \\
\hline 11 & Muro & 10,5 & 6,95 & 70 & 1,51 & 49,4 & 0,97 & 1,096 & 1 & 1,05 & 55,1 & 0,96 & 47,4 & 1,16 \\
\hline 11 & Muro & 13 & 6,95 & 70 & 1,87 & 46,8 & 1,00 & 1,096 & 1 & 1,05 & 53,9 & 0,99 & 46,3 & 1,16 \\
\hline 11 & Muro & 11,2 & 6,95 & 70 & 1,61 & 47,8 & 0,98 & 1,096 & 1 & 1,05 & 53,9 & 0,97 & 46,4 & 1,16 \\
\hline 11 & Muro & 10 & 6,95 & 70 & 1,44 & 45,1 & 0,97 & 1,096 & 1 & 1,05 & 50,3 & 0,95 & 42,8 & 1,18 \\
\hline 11 & Muro & 13,2 & 6,95 & 70 & 1,90 & 44,8 & 1,00 & 1,096 & 1 & 1,05 & 51,6 & 0,99 & 44,4 & 1,16 \\
\hline 11 & Muro & 13,3 & 6,95 & 70 & 1,91 & 44,5 & 1,00 & 1,096 & 1 & 1,05 & 51,2 & 0,99 & 44,1 & 1,16 \\
\hline 11 & Muro & 12,9 & 6,95 & 70 & 1,86 & 48,8 & 1,00 & 1,096 & 1 & 1,05 & 56,2 & 0,99 & 48,3 & 1,16 \\
\hline 11 & Muro & 13,1 & 6,95 & 70 & 1,88 & 43,5 & 1,00 & 1,096 & 1 & 1,05 & 50,1 & 0,99 & 43,1 & 1,16 \\
\hline 11 & Muro & 13 & 6,95 & 70 & 1,87 & 42,5 & 1,00 & 1,096 & 1 & 1,05 & 48,9 & 0,99 & 42,1 & 1,16 \\
\hline & & & & & & & & & & & & Pron & edio & 1,16 \\
\hline
\end{tabular}

\section{REFERENCIAS}

(1) Institute AC (2010). Guide for Obtaining Cores and Interpreting Compressive Strength Results. ACI 2144R-10. Farmington Hills, MI, USA.

(2) Institute BS (2009). BS EN 12504-1:2009. Testing Concrete in Strutures Part 1: Cored specimens - Taking, examining and testing in compression.

(3) Técnicas ABDN (2015). Concreto - Extração, preparo, ensaio e análise de testemunhos de estruturas de concreto Parte 1: Resistência à compressão. NBR 7680:2015. Río de Janeiro, Brasil.

(4) Materials ASfT (2004). Test for obtaining and testing drilled cores and sawed beams of concrete. ASTM C 42/C 42M04. 100 Barr Harbor Drive, PO, USA: ASTM International.

(5) Normalización OND (2015). Norma Cubana NC 724:2015. Ensayos del hormigón - resistencia del hormigón en estado endurecido (ISO 1920-4:2005, MOD).

(6) Grubbs, A. R. (2015). Evaluation of In-Place Concrete Strength by Core Testing. Auburn University.

(7) Helene, P. (2012). Contribuição à análise da resistência do concreto em estruturas existentes para fins de avaliação da segurança. Construindo, 4(01).

(8) Gil Andrés, A. (2011). Evolución de la resistencia del hormigón con la edad y la temperatura.

(9) Pul, S., Husem, M., Arslan, M. E., Zandi, Y. (Eds.) (2011). Investigation of relation between core and cylindrical strength of concrete specimen cured in different conditions. En Proceedings of the 4th WSEAS international conference on Energy and development-environment-biomedicine. World Scientific and Engineering Academy and Society (WSEAS).

(10) Normalización OND (2003). Norma Cubana NC 318: 2003. Hormigón endurecido extracción y preparación de testigos cilíndricos.

(11) Normalización CED (1986). Norma Cubana NC 54-122:1986. Materiales y productos de la construcción hormigón, extracción y preparación de testigos cilíndricos. 